\title{
Prevalence of Errors in Anaphylaxis in Kids (PEAK): A Multi-Center Simulation-Based Study
}

Tensing Maa, MD; Daniel Scherzer, MD²; llana Harwayne-Gidansky, MD, FAAP ${ }^{3}$; Tali Capua, $\mathrm{MD}^{4}$; David O. Kessler, MD, MSc${ }^{5}$; Jennifer L. Trainor, MD ${ }^{6}$; Priti Jani, MD, MPH${ }^{7}$; Becky Damazo, RN, CHSE-A, CPNP-A, MSN ${ }^{8}$; Kamal Abulebda, MD ${ }^{9}$; Maria Carmen G. Diaz, MD, FAAP, FACEP ${ }^{10}$; Rana Sharara-Chami, MD ${ }^{11}$; Sushant Srinivasan, MD, MSc ${ }^{12}$; Adrian Zurca, MD, MEd ${ }^{13}$; Ellen S. Deutsch, MD, $\mathrm{MS}^{14}$

Co-senior authors: Elizabeth A. Hunt, MD, MPH, PhD ${ }^{15}$; Marc Auerbach MD, MSCi ${ }^{16}$ and the PEAK Investigators of the International Network for Simulation-based Pediatric Innovation, Research and Education (INSPIRE)

\footnotetext{
${ }^{1}$ Division of Pediatric Critical Care Medicine, Nationwide Children's Hospital, Ohio State University College of Medicine

${ }^{2}$ Division of Pediatric Emergency Medicine, Nationwide Children's Hospital, Ohio State University College of Medicine

${ }^{3}$ Pediatric Critical Care Medicine, Stony Brook Children's Hospital

${ }^{4}$ Pediatric Emergency Medicine, Dana-Dwek Children's Hospital, Tel Aviv Sourasky Medical Center

${ }^{5}$ Pediatric Emergency Medicine, Columbia University Vagelos College of Physicians and Surgeons

${ }^{6}$ Division of Emergency Medicine, Ann \& Robert H. Lurie Children's Hospital of Chicago, Northwestern University Feinberg School of Medicine

${ }^{7}$ Section of Critical Care, The University of Chicago Comer Children's Hospital,

${ }^{8}$ California State University, Chico

${ }^{9}$ Division of Pediatric Critical Care Medicine, Indiana University School of Medicine and Riley Hospital for Children at Indiana University Health

${ }^{10}$ Division of Emergency Medicine, Nemours/Alfred I. duPont Hospital for Children

${ }^{11}$ Department of Pediatrics and Adolescent Medicine, American University of Beirut

${ }^{12}$ Department of Pediatrics, University of Wisconsin School of Medicine and Public Health 13Division of Pediatric Critical Care, Penn State Hershey Children's Hospital

${ }^{14}$ Department of Anesthesiology and Critical Care, Children's Hospital of Philadelphia, Perelman School of Medicine of the University of Pennsylvania

${ }^{15}$ Departments of Anesthesiology and Critical Care Medicine, Pediatrics and Health Informatics, Johns Hopkins University School of Medicine

${ }^{16}$ Pediatric Emergency Medicine, Yale University School of Medicine
}

Please address correspondence to Tensing Maa, M.D., Nationwide Children's Hospital, Division of Pediatric Critical Care Medicine, 700 Children's Drive, Columbus, Ohio 43205

Tensing.Maa@nationwidechildrens.org; 614-722-3418

(address for reprints)

No financial support was obtained for this study. The authors have no conflicts of interest relevant to this manuscript to disclose.

\author{
Word count, abstract: 272 \\ Word count, text: 3154
}

This is the author's manuscript of the article published in final edited form as:

Maa, T., Scherzer, D. J., Harwayne-Gidansky, I., Capua, T., Kessler, D. O., Trainor, J. L., ... \& Sharara-Chami, R. (2019). Prevalence of Errors in Anaphylaxis in Kids (PEAK): A Multicenter Simulation-Based Study. The Journal of Allergy and Clinical Immunology: In Practice. https://doi.org/10.1016/j.jaip.2019.11.013 
46

47

48

49

50

Abstract

Background: Multi-institutional, international practice variation of pediatric anaphylaxis management by healthcare providers has not been reported.

Objective: Characterize variability in epinephrine administration for pediatric anaphylaxis across institutions, including frequency and types of medication errors.

Methods: A prospective, observational, study using a standardized in situ simulated anaphylaxis scenario was performed across 28 healthcare institutions in six countries. The on-duty healthcare team was called for a child (patient simulator) in anaphylaxis. Real medications and supplies were obtained from their actual locations. Demographic data about team members, institutional protocols for anaphylaxis, timing of epinephrine delivery, medication errors, and systems safety issues discovered during the simulation were collected.

Results: Thirty-seven in situ simulations were performed. Anaphylaxis guidelines existed in $41 \%$ (15/37) of institutions. Teams used a cognitive aid for medication dosing $41 \%(15 / 37)$ of the time and 32\% (12/37) for preparation. Epinephrine auto injectors (EAls) were not available in $54 \%(20 / 37)$ of institutions and were used in only $14 \%$ (5/37) simulations. Median time to epinephrine administration was 95 seconds (IQR 77, 252) for EAI and 263 seconds (IQR 146, 407.5) for manually prepared epinephrine $(p=.12)$. At least one medication error occurred in $68 \%(25 / 37)$ of simulations. Prior nursing experience with epinephrine administration for anaphylaxis was associated with fewer preparation $(p=.04)$ and administration $(p=.01)$ errors. Latent safety threats (LSTs) were reported by $30 \%$ (11/37) of institutions, more than half of these (6/11) involved a cognitive aid.

Conclusion and Relevance: A multicenter, international study of simulated pediatric anaphylaxis reveals: 1) variation in management between institutions in usage of protocols, 
cognitive aids, and medication formularies, 2) frequent errors involving epinephrine, 3) LSTs

related to cognitive aids among multiple sites.

\section{Highlights box: (35 words)}

What is already known about this topic? Factors impacting patient safety in pediatric anaphylaxis management across healthcare institutions are unknown.

74 What does this article add to our knowledge? Preventable medication errors involving epinephrine are more prevalent than previously recognized.

How does this study impact current management guidelines? Variability for use of protocols, cognitive aids, and medication formularies exist.

Keywords: simulation, anaphylaxis, medication error, autoinjector, epinephrine

Abbreviations: EAI (Epinephrine auto injector), El (Epinephrine injection), IM (Intramuscular), IV 


\section{Introduction}

Anaphylaxis is a severe, life threatening, systemic allergic reaction that is rapidly progressive and potentially fatal.(1) In the United States, estimated lifetime prevalence is at least $1.6 \%$ with an increasing incidence globally and in children. (2) Rapid deterioration and death can occur within minutes from the onset of symptoms, and prompt reversal can occur after administration of intramuscular (IM) epinephrine.(3-4) Delays in epinephrine treatment increase the risk of adverse outcomes including mortality. $(5,6)$ The recommended dose and route for treating pediatric anaphylaxis is $0.01 \mathrm{mg} / \mathrm{kg}$ administered IM in the vastus lateralis muscle.(1) IM epinephrine is given via an epinephrine injection (EI) or an epinephrine autoinjector (EAI). El requires that the epinephrine dose be calculated and drawn up from a vial into a syringe whereas EAls deliver a single dose of epinephrine via a disposable, pre-filled, automatic injection device.

A review of the literature from 1990-2015 by Cohen et al. found an extensive list of hazards involving epinephrine use for anaphylaxis.(7) However, only a small number of singlecenter, observational and descriptive studies have reported on errors by healthcare providers associated with El compared to EAI delivery. $(8,9)$ Knowledge and skill gaps in the use of the EAI among healthcare providers have been identified. $(8,10)$ There is no published multi-center overview of clinical or simulation research comparing the prevalence of errors with El versus EAl in the management of anaphylaxis by healthcare providers.(8) Randomized controlled trials of anaphylaxis management are currently not feasible given the life-threatening nature of this condition. Additionally, anaphylaxis has an unpredictable incidence with varied presentations across different clinical disciplines 
105 throughout healthcare systems. Standardized in situ simulation-based assessments have been

106 used to investigate quality of care across a spectrum of healthcare systems without the

107 unpredictability, variability and higher stakes inherent to studying care delivered to actual

108 patients. $(11,12)$ In situ simulation research involves bringing the simulator into the clinical

109 environment to assess the quality of care delivered by intact care teams using real-world

110 equipment.(13) Potential safety concerns, or latent safety threats (LSTs), are often exposed. We

111 utilized simulation-based assessments to examine management of anaphylaxis in pediatric

112 patients across different healthcare institutions.

113 Our primary objective was to characterize variability in practices across institutions

114 related to epinephrine administration, the frequency and types of epinephrine medication

115 errors, and to explore factors associated with errors. We hypothesized that errors would be

116 more likely to occur with El than with EAI and sought to investigate the point prevalence of

117 errors in anaphylaxis management in children during in situ simulation.

118 
119

120

\section{Methods}

\section{Study Design}

This was a prospective, multicenter, international simulation-based study of the management of pediatric anaphylaxis at various patient care locations in healthcare institutions. We report our study in accordance with reporting guidelines for simulation-based research extensions for the STROBE statement.(14) Recruitment of a convenience sample began March 2018 via email and social media (Twitter) advertisement directed to members of the International Network for Simulation-based Pediatric Innovation, Research and Education (INSPIRE), the Pediatrics section of the Society for Simulation in Healthcare (SSH) and the International Pediatric Simulation Society (IPSS). Healthcare institutions that care for pediatric patients capable of performing a single in situ anaphylaxis simulation scenario within a sixmonth period were included. Multiple simulations from a single institution were allowed if they were performed on different clinical units with unique healthcare teams. Each site principal investigator (PI) obtained local institutional review board approval for exempt status.

Institutions unable to obtain approval and accomplish the simulation within the specified time were excluded. As these simulations were performed in the real work environment, feasibility depended on competing clinical demands, thus, a six-month period allowed flexibility in scheduling the simulation.

Setting

Twenty-eight institutions from six countries (Israel, Spain, Lebanon, Germany, New Zealand, and 16 states in the United States) submitted data from 37 simulated events that occurred May 4 through November 20, 2018. Almost all simulations $(n=36)$ were performed in 
141 tertiary or quaternary care academic children's hospitals, ranging in size from 30 to over 300

142 pediatric beds, and $81 \%(n=30)$ cared soley for pediatric patients. Only one simulation was

143 performed in the emergency department (ED) of a community hospital. Three institutions (11\%)

144 performed the scenario in more than one clinical location. Simulations took place most often in

145 the ED (38\%), followed by the inpatient non-oncology floor (30\%) and the intensive care unit

146 (ICU) (16\%). Other locations included an infusion center, procedural sedation unit and oncology

147 clinic (see Table I).

148

150

\section{Simulation Scenario}

The scenario, created by a multidisciplinary group of simulation experts, involved a five year-old, 20 kilogram $(\mathrm{kg})$ child with a history of peanut and drug allergies who has an anaphylactic reaction after receiving an intravenous (IV) medication. The human patient simulator manikin had an IV catheter in place at the start of the simulation and demonstrated clinical symptoms of anaphylaxis as allowed by the individual simulator. The scenario ended after epinephrine was administered. The procedures for selection of the in situ environment and delivery of the simulation (including pre-simulation script and post-simulation debriefing) were standardized (see eMethods in the Online Repository). The following components of the simulation were site specific and not standardized: brand of simulator, IV medication causing the allergic reaction, use of video recording, pre-announcement of the simulation to participants, team composition, and team leader.

Site PIs were directed to choose an in situ environment that would allow the on duty healthcare team to respond. The scenario started with calling the team for help with a patient in anaphylaxis, thus obviating the need to recognize and diagnose an allergic drug reaction. 
163

164

165

166

167

168

Participants managed the anaphylaxis scenario as they would in real life. Real medications and supplies for the administration of epinephrine were obtained from their authentic locations in the clinical space. Immediately after the simulation, the team participated in a postperformance debriefing focused on adherence to anaphylaxis guidelines, drug dosing and administration errors, as well as the identification of systems, patient or staff safety concerns.

\section{Data collection}

Demographic data were obtained to characterize the simulation team and understand the local standards for anaphylaxis and epinephrine administration. Data collected using a standardized form included: site demographics (availability of EAI and in which locations, existence of local anaphylaxis guidelines), dose, concentration, route, correct site of administration and the timing of epinephrine delivery. This data was provided by the site PI. Time dependent metrics were verified after reviewing video recordings when available. Data was entered into a central server, associated with the SSH International Simulation Data Registry, over a web-based, password protected collection tool. Published anaphylaxis guidelines for dosing were used to define correct dose, and for a $20 \mathrm{~kg}$ child this is the 0.15 milligram $(\mathrm{mg}$ ) autoinjector or $0.01 \mathrm{mg} / \mathrm{kg}$, i.e. $0.2 \mathrm{mg} .(1)$ The correct concentration is $1: 1000$ or $1 \mathrm{mg} / \mathrm{milliliter}(\mathrm{ml})$ for El. The correct route is IM. It was also considered a separate error if specifics on dose, concentration or route were not prescribed (i.e. the practitioner only said "Let's give epi."). The site PI was instructed to position themselves so they could observe the medication preparation process to ensure correct volume and concentration was drawn up. An administration error for EAl use was recorded if the device was not held in place for 3 seconds. (15) Medication errors in prescribing (dose, concentration, 
185

186

187

188

189

190

192

193

route), preparation (dose and concentration) or administration (route or location), as well as safety issues discovered during the simulation were collected. An error in prescribing that was prepared and given as incorrectly prescribed could potentially be counted as 3 separate errors (one for prescribing, one for preparation and one for administration). This was because there was opportunity for the error to be caught and corrected at each of those time points.

\section{Statistical Analysis}

Data were summarized using frequency and percentage for categorical variables and medians with interquartile range (IQR) for continuous. Demographic and simulation team factors associated with medication errors were evaluated using odds ratio (OR) and $95 \%$ confidence intervals $(\mathrm{Cl})$ were assessed using univariate logistic regression with Firth's penalized likelihood for small sample size. Differences in time to order and time to administration of epinephrine were assessed using Wilcoxon rank sum tests. Primary analysis includes all simulation events from each site. Within-site correlation was not accounted for due to limitations of sample size. Sensitivity analysis using one simulation event per site was performed and results were substantively similar (not shown). All analyses were conducted using SAS 9.4 (SAS Institute, Cary, NC) with two-sided p-values $<0.05$ considered statistically significant. 
203

204

205

206

207

208

209

210

211

212

213

214

215

216

217

218

219

220

222

\section{Results}

Table I lists simulation site and team characteristics. Teams included at least one nurse and practitioner (physician or advanced practice nurse/physician assistant) for all simulations. An EAI was used in five (14\%) simulations. Only 46\% (17/37) of simulations reported having an EAI available somewhere in their institution, not necessarily throughout all locations. The sites in Israel, Lebanon and New Zealand do not have any epinephrine autoinjectors available in their hospitals. The sites in Spain used Jext ${ }^{\circledast}$ and the United States and Germany used EpiPen ${ }^{\circledR}$. Institutions using EAI as first-line therapy in some, but not all, areas of the hospital stocked them mostly within medication dispensing units (47\%) and in radiology (41\%). Table E1 in the online repository has specifics about which locations EAI are available as first line therapy and how they are stored.

\section{Epinephrine errors}

At least one error was made in $68 \%$ (25/37) of simulations. Of the five events using EAl: $40 \%(n=2)$ of errors were due to over-dosing, $20 \%(n=1)$ included an error in administration (i.e. injection duration less than 3 seconds per recommendations).(15) Of the 32 events utilizing El: $53 \%(n=17)$ involved an error only in prescribing, $16 \%(n=5)$ had an error only in preparation and $9 \%(n=3)$ had separate errors made during both prescribing and preparation. Figure 1 shows the progression and propagation of El and EAl errors. In two events, the medication dose, concentration, and route (i.e., all 3 parameters) were not specified. Epinephrine was ordered to be administered IV in $24 \%(n=9)$. A specific intramuscular location for giving epinephrine was not specified in 43\% (16/37), although we did not count this as an error. Figure 2 is a Pareto chart showing types of errors in descending order of frequency. There were 
five "near miss" events involving a prescribing error that was caught and did not reach the patient.

\section{System-level safety hazards}

Nearly one-third of sites $(n=11)$ discovered one or more safety hazards during

simulations. More than half of these $(n=6,54 \%)$ involved problems with usability or design of a cognitive aid, such as missing anaphylaxis dosing, dosing only listed in milligrams and not milliliters, cardiac arrest dosing used, and conflicting dosing recommendations. Table E2 in the Online Repository contains additional details related to latent safety threats.

Risk factors for errors

Estimated odds ratios for presence of at least one medication error by site and team characteristics are shown in Table II. Teams that had a nurse with prior experience giving epinephrine for anaphylaxis were significantly associated with events without medication preparation errors $(\mathrm{OR}=0.2,95 \% \mathrm{Cl}=[0.04,0.93], \mathrm{p}=.04)$ and with events without administration errors $(\mathrm{OR}=0.13,95 \% \mathrm{Cl}=[0.03,0.62], \mathrm{p}=.01)$. There were an insufficient number of sites using EAI to compare error rates with El in a meaningful way.

Table III compares times from medication ordering to administration for El to EAl use. Although the difference was not statistically significant, the median time to administration between the two forms of epinephrine was over 2.5 minutes longer for El. Time to prepare the EAI dose was significantly shorter $(p=.02)$ than for El. 


\section{Discussion:}

This is the first, multi-center prospective, observational study to investigate the variability and vulnerabilities of pediatric anaphylaxis management in healthcare institutions, many of which are academic, pediatric medical centers. The primary objective was to characterize variability in practices across institutions related to epinephrine administration, the frequency and types of epinephrine medication errors, and to explore factors associated with errors. The key results of this study are that, in this cohort, errors in anaphylaxis management are common in clinical sites specialized in pediatric acute or critical care. High rates of both prescribing errors and IV administration were observed during the management of a simulated child with anaphylaxis. The use of an EAI and the presence of an anaphylaxis guideline have the potential to improve safety but both were uncommon in this cohort and not associated with reduced error. $(7,16)$ Prior nursing experience giving epinephrine for anaphylaxis was the only protective factor against preparation and administration errors. At least one medication error was made in $68 \%$ of events. Medication dosing in pediatrics is weight-based and more complex than for adults, thus most pediatric medication errors occur during the prescribing stage and are related to dosing errors $(17,18)$. In this study, the most common errors centered on incorrect prescribing of epinephrine, which occurred with both EAI and El, followed by errors in El preparation. Prescribing errors that were not caught resulted in the administration of epinephrine via the IV route in $24 \%$ of cases that used El. The errors noted in this cohort pose a significant potential for harm due to the narrow therapeutic index and systemic cardiovascular side effects of epinephrine.(19) Variations in indications for epinephrine use, concentration, routes of administration, commercial delivery devices and 
nomenclature to express dosages and concentrations all contribute to potential for error. (20-

Standardization of the administration of epinephrine through the use of EAl or storing pre-filled syringes of epinephrine specifically designated for IM use in anaphylaxis have the potential to reduce errors. $(7,21)$ Only two sites reported EAl as first line therapy throughout their institution whereas an additional 15 sites have EAI available in select locations. Only 29\% (5/17) of teams with access to an EAI chose to use one to respond to a simulated case of anaphylaxis. Fifty-four percent of sites do not have EAI available at their institution at all. The small number of institutions using EAI precluded identification of a statistically significant difference in error rates compared with El. We observed that EAl use was associated with an almost 3-minute reduction in the median time to administer epinephrine compared to the median time of 4.4 minutes to administration of El. This delay in treatment is concerning as anaphylaxis can lead to death in less than five minutes. (3) Increased costs, drug shortages and systems.(23) As these barriers may not change quickly, it is important to have a mechanism to examine prevalence of epinephrine errors across institutions, understand their causes, and share successful risk mitigation plans in order to prevent patient harm. Organization (WAO) guidelines recommend having a written protocol for management of anaphylaxis.(16) A clinical guideline is of particular use for anaphylaxis, an event that is low

287 frequency per practitioner, and may cause confusion to practitioners about appropriate 
288

289

practice. $(24,25)$ The presence of an identified guideline was not significantly associated with decreased frequency of errors in this study.

There was low overall use of cognitive aids in this study with only $41 \%$ of simulation teams utilizing aids for prescribing and $32 \%$ for preparing medication doses. Cognitive aids, which may include checklists, flowcharts, and posters, can improve the speed and accuracy of task completion, including improving outcomes and decreasing the number of errors in emergency situations.(26) There was no significant association between cognitive aid use and decreased frequency of error in our study.

Cognitive aids are widely underused as they require good design, easy access, and a supportive systemic environment.(27) More than half of the latent safety threats reported in this study involved errors found in institutional cognitive aids themselves. Hazards included missing anaphylaxis dosing on the aids, dosages listed in milligrams but not milliliters (thus requiring hand-calculation of the volume), practitioners selecting cardiac arrest instead of anaphylaxis dosages, conflicting dosage recommendations, and an outdated cognitive aid with the incorrect suggested route (subcutaneous) of administration. In these instances, the design of the cognitive aid itself created a vulnerability for errors.

Prior nursing experience administering epinephrine for anaphylaxis was significantly associated with simulations that were free of medication preparation and administration errors. In emergent situations, nurses are the ones who typically prepare and administer medications and thus play a key role in catching and preventing prescribing errors. (28) Previously reported knowledge and experience gaps of the prescribing practitioner as well as 
the medication nurse may be important contributors to error during management of anaphylaxis and key targets for intervention. $(29,30)$

We have several recommendations to improve anaphylaxis management and patient safety based on our findings. Systems-based interventions could include establishment, dissemination and education of a written guideline or protocol for anaphylaxis management as recommended by the WAO. Additionally, institutions should regularly review their cognitive aids to ensure accessibility, clarity and accuracy. Less than one third of institutions with EAI available used them during these simulations. Use of an EAI, or having an experienced nurse on site might result in decreased error rates and more timely preparation and appropriate administration of epinephrine for anaphylaxis. Additional training using interprofessional anaphylaxis simulations could focus on 1) awareness of the high risk of medication errors, 2) familiarization with cognitive aids 3) use of aids for preparation and administration of epinephrine and 4) exploration of barriers to EAI use in institutions with EAI available. This study has several limitations. Limitations intrinsic to simulation-based studies include the fact that simulated scenarios may not fully mimic real life, which may impact the speed and efficiency of health care providers' actions. To mitigate this, we used an in situ simulation and instructed sites to approximate an authentic response as much as possible, including use of real medications and equipment. Simulation studies run the risk of potential unmeasured confounders including deviations in conducting the scenario, differences in team composition, and practice variation over time. We attempted to mitigate these by using a very simple, standardized scenario and limiting the time period for its performance. Generalizability may be limited as this study was performed mostly in academic pediatric institutions with 
simulation programs in North America and may not be as relevant in institutions with different attributes. underpowered to detect statistically significant differences in errors between EAI and El. However, it provides preliminary pilot data on the proportion of EAI use at institutions and the error rate with El to understand what sample size is needed for an appropriately powered 337 study.

\section{Conclusion} anaphylaxis confirms wide variability among healthcare institutions for usage of protocols,

341 cognitive aids, and medication formularies, timing of epinephrine dose delivery, and types of

342 medication errors made. Prior nursing experience with giving epinephrine for anaphylaxis was

343 significantly associated with fewer preparation and administration errors. There was an 344 unexpectedly low rate of EAI use, and not all teams with access to EAls used them.

\section{Acknowledgements}

347 The authors would like to acknowledge the contributions of the International Network for

348 Simulation-Based Pediatric Innovation, Research, \& Education (INSPIRE) who have helped to 349 shape this project and the International Meeting for Simulation in Healthcare for providing 350 conference meeting space. 
Group information: The International Network for Simulation-Based Pediatric Innovation,

Research, \& Education (INSPIRE) PEAK Investigators were the authors and those collaborators

354 listed below.

Site collaborators and data collection: Joo Lee Song, MD, Children's Hospital Los Angeles; Robyn

Wing, MD, MPH, Hasbro Children's Hospital; Susan Teman, BSN, RN, Helen DeVos Children's

Hospital; Antonio Rodriguez-Nunez, MD, PhD, Hospital Clinico Universitario de Santiago; Carisa

Schneider, MD, Jacobi Medical Center; Danielle Mercurio, DO, Johns Hopkins All Children's

Hospital; Christie Gutierrez, MD, Columbia University Vagelos College of Physicians and

Surgeons; Michelle Gaba, MD, Mount Sinai-Beth Israel; Benny L. Joyner, Jr., MD, MPH,

Children's Hospital, University of North Carolina; Elizabeth S. Vukin, MD, and Jared Henricksen,

MD, Primary Children's Hospital; Lynda Knight, RN, MSN, Stanford Children's Health; Trish

Wood, RN and Renee England, RN, Starship Children's Health; Christina Cochran, MD,

Contributor to conceptualization and design, administrative, or technical support: Aaron

Calhoun, MD, University of Louisville; Matt Kurrek, MD, FRCP(C), University of Toronto;

369 Elizabeth Biddell, MD, Akron Children's Hospital; Jennifer Arnold, MD, MSc, Johns Hopkins All

370 Children's Hospital; Melinda F. Hamilton, MD, MS, UPMC Children's Hospital of Pittsburgh;

371 Nancy Tofil, MD, University of Alabama at Birmingham

372 Figure preparation: William C. Ray, PhD, The Ohio State University Biophysics Program, Battelle

373 Center for Mathematical Medicine, The Research Institute at Nationwide Children's Hospital 
374 Statistical Analysis: Melissa Moore-Clingenpeel, MAS, The Research Institute at Nationwide

375 Children's Hospital For personal use only. No other uses without permission. Copyright @2019. Elsevier Inc. All rights reserved. 


\section{References}

1. Sampson HA, Munoz-Furlong A, Campbell RL, Adkinson NF, Jr., Bock SA, Branum A, et al. Second symposium on the definition and management of anaphylaxis: summary report-Second National Institute of Allergy and Infectious Disease/Food Allergy and Anaphylaxis Network symposium. J Allergy Clin Immunol. Feb 2006;117(2):391-397.

2. Wood RA, Camargo CA, Jr., Lieberman P, Sampson HA, Schwartz LB, Zitt M, et al. Anaphylaxis in America: the prevalence and characteristics of anaphylaxis in the United States. J Allergy Clin Immunol. Feb 2014;133(2):461-467.

3. Pumphrey RS, Roberts IS. Postmortem findings after fatal anaphylactic reactions. $J$ Clin Pathol. Apr 2000;53(4):273-276.

4. Greenhawt M, Gupta RS, Meadows JA, Pistiner M, Spergel JM, Camargo CA, Jr., et al. Guiding Principles for the Recognition, Diagnosis, and Management of Infants with Anaphylaxis: An Expert Panel Consensus. J Allergy Clin Immunol Pract. Feb 52019.

5. Dhami S, Panesar SS, Rader T, Muraro A, Roberts G, Worm M, et al. The acute and longterm management of anaphylaxis: protocol for a systematic review. Clin Transl Allergy. Apr 10 2013;3(1):14.

6. Muraro A, Dubois AE, DunnGalvin A, Hourihane JO, de Jong NW, Meyer R, et al. EAACI Food Allergy and Anaphylaxis Guidelines. Food allergy health-related quality of life measures. Allergy. Jul 2014;69(7):845-853.

7. Cohen MB, Saunders SS, Wise SK, Nassif S, Platt MP. Pitfalls in the use of epinephrine for anaphylaxis: patient and provider opportunities for improvement. Int Forum Allergy Rhinol. Mar 2017;7(3):276-286.

8. Chime NO, Riese VG, Scherzer DJ, Perretta JS, McNamara L, Rosen MA, et al. Epinephrine Auto-Injector Versus Drawn Up Epinephrine for Anaphylaxis Management: A Scoping Review. Pediatr Crit Care Med. Aug 2017;18(8):764-769.

9. Campbell RL, Bellolio MF, Knutson BD, Bellamkonda VR, Fedko MG, Nestler DM, et al. Epinephrine in anaphylaxis: higher risk of cardiovascular complications and overdose after administration of intravenous bolus epinephrine compared with intramuscular epinephrine. J Allergy Clin Immunol Pract. Jan-Feb 2015;3(1):76-80.

10. Scherzer DJ, Chime NO, Tofil NM, Hamilton MF, Singh K, Stanley RM, et al. Survey of pediatric trainee knowledge: dose, concentration, and route of epinephrine. Ann Allergy Asthma Immunol. Apr 2017;118(4):516-518.

11. Weinger MB. The pharmacology of simulation: a conceptual framework to inform progress in simulation research. Simul Healthc. Feb 2010;5(1):8-15.

12. Cheng A, Auerbach M, Hunt EA, Chang TP, Pusic M, Nadkarni V, et al. Designing and conducting simulation-based research. Pediatrics. Jun 2014;133(6):1091-1101.

13. Patterson MD, Geis GL, LeMaster T, Wears RL. Impact of multidisciplinary simulationbased training on patient safety in a paediatric emergency department. BMJ Qual Saf. May 2013;22(5):383-393.

14. Cheng A, Kessler D, Mackinnon R, Chang TP, Nadkarni VM, Hunt EA, et al. Reporting Guidelines for Health Care Simulation Research: Extensions to the CONSORT and STROBE Statements. Simul Healthc 2016;11(4):238-248. 
15. How to use an EpiPen ${ }^{\circledast}$ (epinephrine injection, USP) Auto-Injector. Morgantown, WV: Mylan Specialty LP; 2018. Available at: https://www.epipen.com//media/files/epipen/howtouseepipenautoinjector.pdf. Accessed July 31, 2019.

16. Simons FE, Ebisawa M, Sanchez-Borges M, Thong BY, Worm M, Tanno LK, et al. 2015 update of the evidence base: World Allergy Organization anaphylaxis guidelines. World Allergy Organ J. 2015;8(1):32.

17. Kaushal R, Bates DW, Landrigan C, McKenna KJ, Clapp MD, Federico F, et al. Medication errors and adverse drug events in pediatric inpatients. JAMA. 2001;285:2114-2120.

18. Fortescue EB, Kaushal R, Landrigan C, McKenna KJ, Clapp MD, Federico F, et al. Prioritizing strategies for preventing medication errors and adverse drug events in pediatric inpatients. Pediatrics. 2003;111(4 Pt 1):722-9.

19. Advis PPS. An Update on the "Epi"demic: Events Involving EPINEPHrine. Pa Patient Saf Advis. 2009;6(3):102-103.

20. Advis PPPS. Let's stop this "Epi" demic! Preventing errors with epinephrine. 3. 2006;(3)(16-17).

21. Kanwar M, Irvin CB, Frank JJ, Weber K, Rosman H. Confusion about epinephrine dosing leading to iatrogenic overdose: a life-threatening problem with a potential solution. Ann Emerg Med. Apr 2010;55(4):341-344.

22. Rolfe S, Harper NJ. Ability of hospital doctors to calculate drug doses. BMJ. May 6 1995;310(6988):1173-1174.

23. Sargel CL, Maa T. Epinephrine Auto-Injectors Versus Manually Drawn Up Epinephrine: Is There a Better Option? Pediatr Crit Care Med. Aug 2017;18(8):807-808.

24. Hughes $G$, Fitzharris P. Managing acute anaphylaxis. New guidelines emphasise importance of intramuscular adrenaline. BMJ. Jul 3 1999;319(7201):1-2.

25. Woolf SH, Grol R, Hutchinson A, Eccles M, Grimshaw J. Clinical guidelines: potential benefits, limitations, and harms of clinical guidelines. BMJ. Feb 20 1999;318(7182):527530.

26. Goldhaber-Fiebert SN, Howard SK. Implementing emergency manuals: can cognitive aids help translate best practices for patient care during acute events? Anesth Analg. Nov 2013;117(5):1149-1161.

27. Burian BK, Clebone A, Dismukes K, Ruskin KJ. More Than a Tick Box: Medical Checklist Development, Design, and Use. Anesth Analg. Jan 2018;126(1):223-232.

28. Hughes RG, Edgerton EA. Reducing pediatric medication errors: Children are especially at risk for medication errors. Am J Nurs. May 2005;105(5):79-80.

29. Johnston EB, King C, Sloane PA, Cox JW, Youngblood AQ, Lynn Zinkan J, et al. Pediatric anaphylaxis in the operating room for anesthesia residents: a simulation study. Paediatr Anaesth. Feb 2017;27(2):205-210.

30. Sanko, JS, Mckay, M. Impact of Simulation-Enhanced Pharmacology Education in Prelicensure Nursing Education. Nurse Educ. Sep/Oct 2017;42(5S), S32-S37. 
460 Figure 1: Pathways for error propagation during 32 simulation events using manually drawn up

461 epinephrine (1a) and five events with epinephrine autoinjectors (1b). Simulation-discoverable

462 medication errors and opportunities for correction differ between these formulations. $\mathrm{Rx}=$

463 prescribed, Med Prep $=$ medication preparation, $\mathrm{Admin}=$ administration, $\mathrm{Pt}=$ patient, $\mathrm{Tx}=$

464 treatment

465

466 Figure 2: Pareto chart with decreasing frequency of epinephrine errors from 37 simulations.

467 Some had more than one error. Prescribing of concentration and route and preparation errors

468 are not applicable for epinephrine autoinjectors. Admin $=$ administration, conc $=$ concentration,

469 IV = intravenous.

470

471

472

473

474

475 


\begin{tabular}{|c|c|c|}
\hline \multicolumn{3}{|l|}{ Table I: Simulation and Site Characteristics $(n=37)$} \\
\hline & $\mathrm{N}$ & $\%$ \\
\hline \multicolumn{3}{|l|}{ Institution Specialty } \\
\hline Pediatric & 30 & 81 \\
\hline Mixed (adult and pediatric) & 7 & 19 \\
\hline \multicolumn{3}{|l|}{ Anaphylaxis protocol/guideline } \\
\hline No/Unsure & 22 & 59 \\
\hline Yes & 15 & 41 \\
\hline \multicolumn{3}{|l|}{ Institution allows use of patient's $\mathrm{EAI}^{\mathrm{a}}$} \\
\hline No/Unsure & 21 & 57 \\
\hline Yes & 16 & 43 \\
\hline \multicolumn{3}{|l|}{$\mathrm{EAl}^{\mathrm{a}}$ available somewhere in institution } \\
\hline No/Unsure & 20 & 54 \\
\hline Yes & 17 & 46 \\
\hline \multicolumn{3}{|l|}{$\mathrm{EAl}^{\mathrm{a}}$ as first line therapy throughout institution $(\mathrm{n}=17)$} \\
\hline 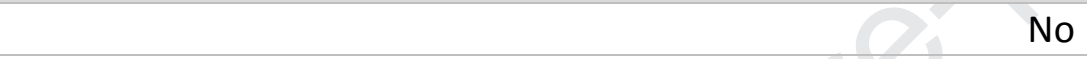 & 15 & 88 \\
\hline Yes & 2 & 12 \\
\hline \multicolumn{3}{|l|}{$\mathrm{EAI}^{\mathrm{a}}$ as first line therapy in some areas of hospital $(n=15)$} \\
\hline No/Unsure & 4 & 27 \\
\hline 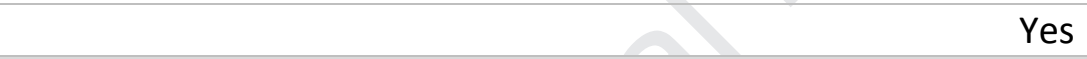 & 11 & 73 \\
\hline \multicolumn{3}{|l|}{ Simulation Location } \\
\hline Emergency Department & 14 & 38 \\
\hline$I C U^{b}$ & 6 & 16 \\
\hline Inpatient floor (Non-Oncology) & 11 & 30 \\
\hline Other & 6 & 16 \\
\hline \multicolumn{3}{|l|}{ Team composition: } \\
\hline Resident & 27 & 73 \\
\hline Fellow & 9 & 24 \\
\hline Attending & 11 & 30 \\
\hline Advance Practice Nurse/Physician Assistant & 6 & 16 \\
\hline Pharmacist & 3 & 8 \\
\hline \multicolumn{3}{|l|}{ Experience of team leader } \\
\hline 5 or more years of clinical experience (after residency) & 7 & 19 \\
\hline Less than 5 years of clinical experience (after residency) & 9 & 24 \\
\hline trainee (resident) & 21 & 57 \\
\hline \multicolumn{3}{|l|}{ Nursing experience } \\
\hline Never given epinephrine for anaphylaxis & 12 & 32 \\
\hline Given $\mathrm{El}^{\mathrm{C}}$ for anaphylaxis at least once & 17 & 46 \\
\hline Given EAl $^{\mathrm{a}}$ for anaphylaxis at least once & 8 & 22 \\
\hline Cognitive aid used for prescribing & 15 & 41 \\
\hline
\end{tabular}


Cognitive aid used for preparation

${ }^{\mathrm{a}} \mathrm{EAI}=$ Epinephrine auto injector, ${ }^{\mathrm{b}} \mathrm{ICU}=$ Intensive care unit ${ }^{\mathrm{C}} \mathrm{El}=$ Epinephrine injection 
477 Table II: Odds ratio estimates for risk factors associated with epinephrine error

\begin{tabular}{|c|c|c|c|c|c|c|c|c|c|}
\hline \multirow[t]{2}{*}{ Risk Factor } & \multicolumn{3}{|c|}{ Prescribing Error } & \multicolumn{3}{|c|}{ Preparation Error } & \multicolumn{3}{|c|}{ Administration Error } \\
\hline & $\mathrm{OR}^{\mathrm{a}}$ & $95 \% \mathrm{Cl}^{\mathrm{b}}$ & $\begin{array}{c}\mathrm{p}- \\
\text { value }\end{array}$ & OR & $95 \% \mathrm{Cl}$ & $\begin{array}{c}\mathrm{p}- \\
\text { value }\end{array}$ & OR & $95 \% \mathrm{Cl}$ & $\begin{array}{c}\mathrm{p}- \\
\text { value }\end{array}$ \\
\hline $\begin{array}{l}\text { Nursing with prior epinephrine } \\
\text { administration for anaphylaxis }\end{array}$ & 0.65 & $(0.15,2.78)$ & 0.56 & 0.2 & $(0.04,0.93)$ & 0.04 & 0.13 & $(0.03,0.62)$ & .01 \\
\hline $\begin{array}{l}\text { Other Location vs. Intensive } \\
\text { Care Unit }\end{array}$ & 3.18 & $(0.46,22.22)$ & .24 & 5.13 & $(0.58,45.45)$ & .14 & 1.02 & $(0.15,7.09)$ & .99 \\
\hline $\begin{array}{l}\text { Other Location vs. Emergency } \\
\text { Department }\end{array}$ & 1.77 & $(0.42,7.46)$ & .44 & 2.42 & $(0.57,10.31)$ & .23 & 1.32 & $(0.29,5.99)$ & .72 \\
\hline Inexperienced team lead $^{\mathrm{c}}$ & 2.01 & $(0.50,8.13)$ & .33 & 2.93 & $(0.66,13.11)$ & .16 & 2.84 & $(0.55,14.71)$ & .21 \\
\hline $\begin{array}{l}\text { Less than } 3 \text { anaphylaxis } \\
\text { simulations/year }\end{array}$ & 1.8 & $(0.47,6.90)$ & .39 & 1.11 & $(0.29,4.26$ & .88 & 2.25 & $(050,10.10)$ & .29 \\
\hline No attending on team & 2.62 & $(0.61,11.24)$ & .19 & 1.44 & $(0.34,6.10)$ & .63 & 2.42 & $(0.46,12.66)$ & .30 \\
\hline No pharmacist on team & 2.1 & $(0.18,23.91)$ & .55 & 1.32 & $(0.12,15.09)$ & .82 & 3.89 & $(0.12,127.78)$ & .44 \\
\hline 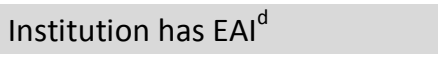 & 3.89 & $(0.33,45.61)$ & .28 & 1.4 & $(0.12,16.43)$ & .79 & 0.41 & $(0.04,4.33)$ & .46 \\
\hline EAl used & 0.61 & $(0.17,2.22)$ & .46 & 0.36 & $(0.09,1.41)$ & .14 & 0.79 & $(0.20,3.15)$ & .74 \\
\hline Cognitive aid used for dosing & 1.73 & $(0.44,6.81)$ & .44 & 0.91 & $(0.24,3.51)$ & .89 & 0.87 & $(0.21,3.77)$ & .87 \\
\hline $\begin{array}{l}\text { Cognitive aid used for } \\
\text { preparation }\end{array}$ & 0.94 & $(0.23 .3 .90)$ & .93 & 1.07 & $(0.28,4.44)$ & .92 & 0.21 & $(0.03,1.50)$ & .12 \\
\hline Protocol/Guideline & 2.27 & $(0.59,8.82)$ & .24 & 1.93 & $(0.51,7.34)$ & .33 & 1.74 & $(0.43,6.95)$ & .43 \\
\hline Pediatric (vs mixed) institution & 1.67 & $(0.32,8.75)$ & .55 & 0.53 & $(0.10,2.77)$ & .45 & 1.13 & $(0.19,6.61)$ & .89 \\
\hline
\end{tabular}

$478{ }^{\mathrm{a}} \mathrm{OR}=$ odds ratio, ${ }^{\mathrm{b}} \mathrm{Cl}=$ confidence interval. ${ }^{\mathrm{C}}$ Inexperienced team lead = still in residency

479 training, $\mathrm{EAI}^{\mathrm{d}}=$ epinephrine auto injector 
Table III: Comparison of time (in seconds) by form of epinephrine used during simulation

\begin{tabular}{|c|c|c|c|c|c|}
\hline & \multicolumn{2}{|c|}{ Drawn up Epi ${ }^{a}(n=32)$} & \multicolumn{2}{|c|}{ Epi ${ }^{\mathrm{a}}$ Auto injector $(n=5)$} & \multirow[b]{2}{*}{$\mathrm{p}$-value } \\
\hline Time from & Median & $I Q R^{b}$ & Median & IQR & \\
\hline Start to order ${ }^{c}$ & 91.5 & $(37.5,227.5)$ & 49 & $(38,184)$ & .74 \\
\hline Order to administration & 114 & $(62,174.5)$ & 28 & $(26,68)$ & .02 \\
\hline Start to administration ${ }^{d}$ & 262.5 & $(146,407.5)$ & 95 & $(77,252)$ & .12 \\
\hline
\end{tabular}

Note: ${ }^{\mathrm{a}} \mathrm{Epi}=$ epinephrine, ${ }^{\mathrm{b}} \mathrm{IQR}=$ interquartile range, Start time $=$ when the team engages in the scenario, just after they are told the patient is in anaphylaxis from an intravenous medication. 'Start to order = the time to when the practitioner finishes verbally ordering the epinephrine medication. ${ }^{d}$ Start to administration = the time to when the epinephrine was administered, includes medication preparation 

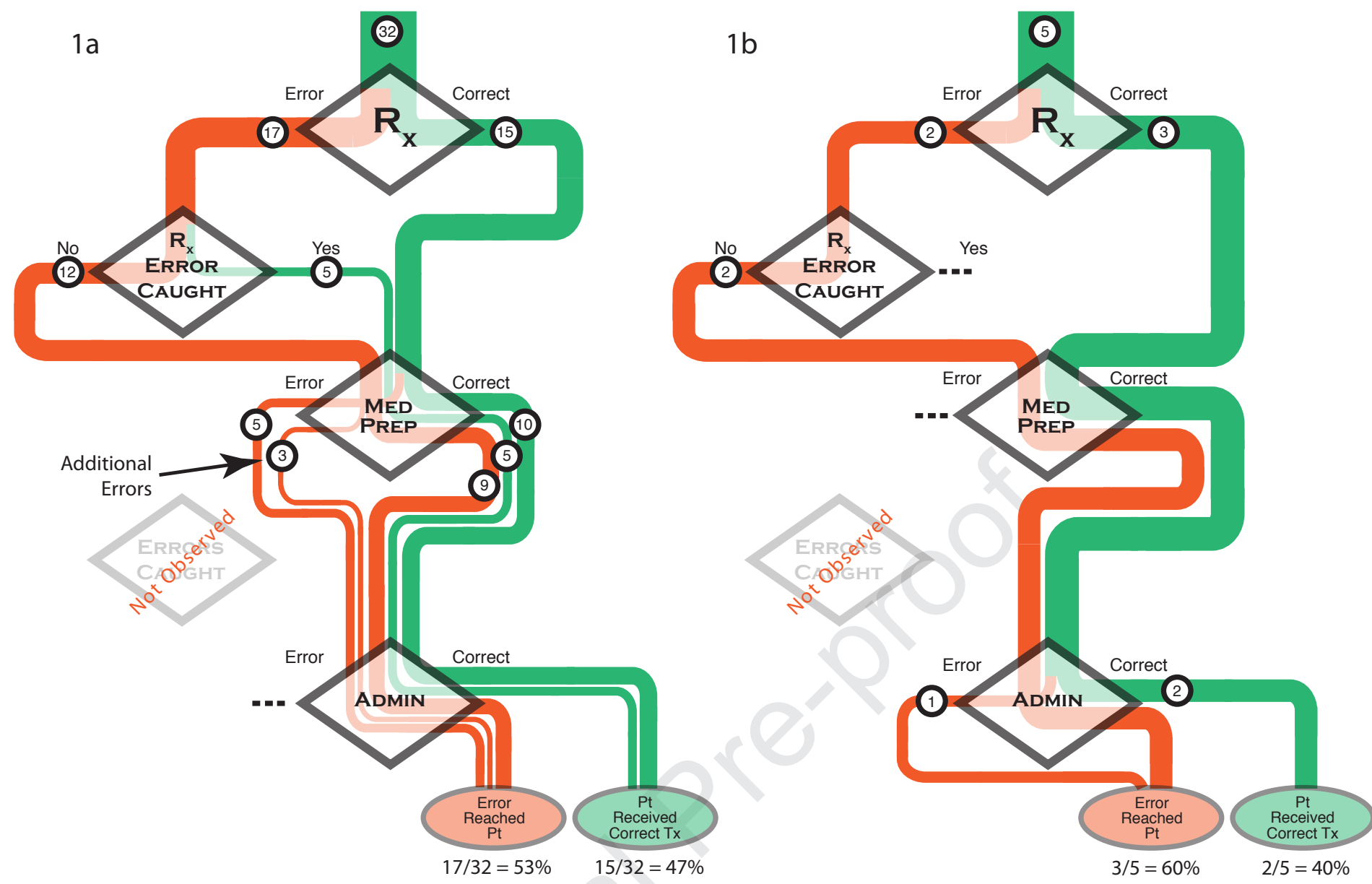


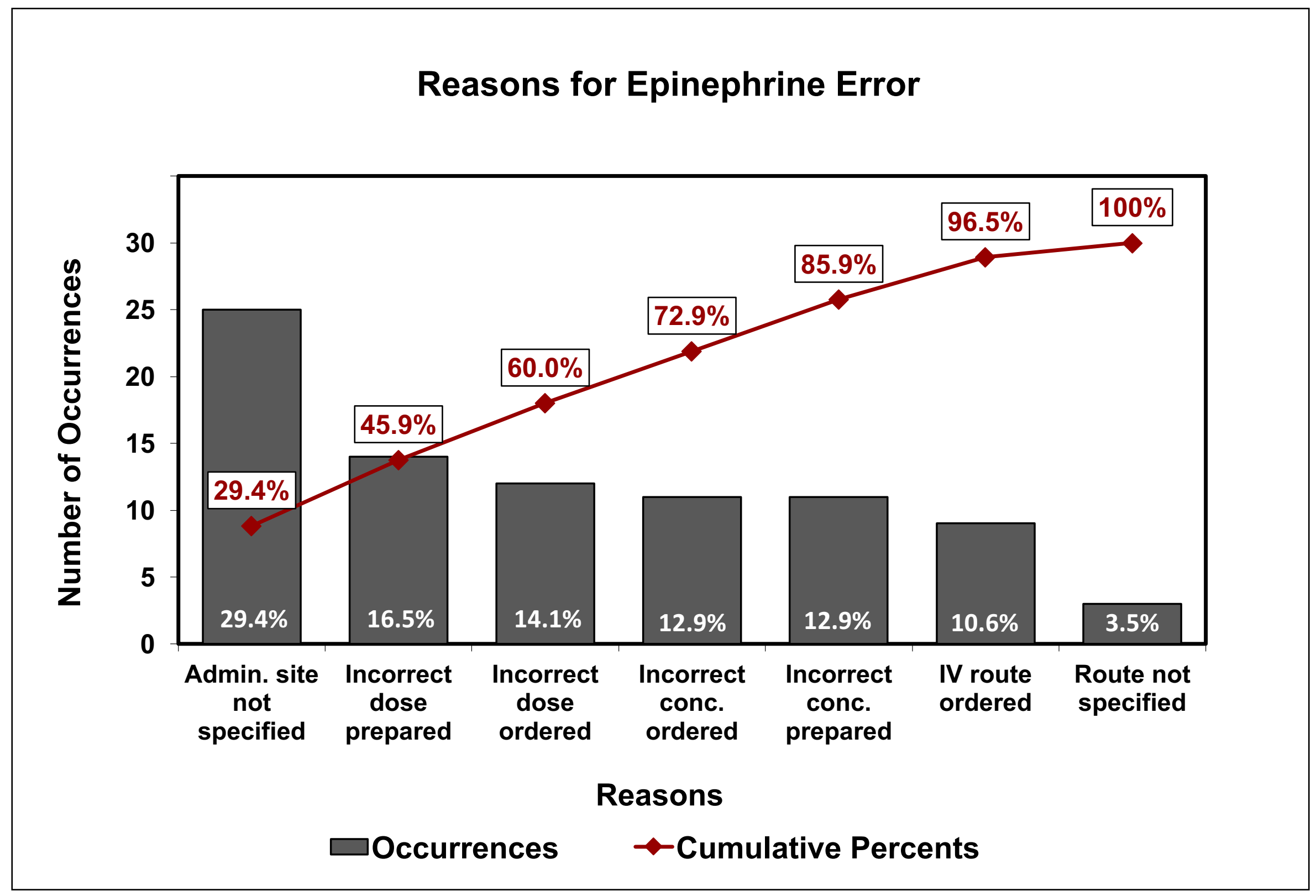


eMethods: Simulation scenario instructions

\section{Choosing which clinical site to perform your simulation}

We ask that the scenario be performed "in situ", and not in a simulation center, because we are interested in recreating and capturing what happens in real life. You can choose which clinical environment to perform the scenario- the ED, ICU, radiology, inpatient floor, etc. I would base your choice on which location will be most receptive to you coming to run a simulation that meets these requirements:

1) We would like to use a healthcare team that is "on duty" and most representative of the team that would normally respond and manage a patient in anaphylaxis on that unit. This will depend on your location. For my hospital, typically a resident, nurse practitioner or fellow and a nurse would manage such a patient. Perhaps an attending would also be involved. I would not want a team of just medical students because that is not a realistic representation for my institution.

There may be some sites that are less accustomed to running a simulation in the real clinical environment. If this is the case, you may want to recruit a healthcare team to participate or provide a prebrief so that the team knows to behave as they would in real life. There will be a question on the data collection survey that asks if this simulation was partially announced or unannounced.

2) We would like to use real medications. So, the staff participating in the simulation should go to where epinephrine is stored on the unit and obtain it from there for med preparation. On some units, this may be an epi auto-injector that is kept in the medication room. Other units may open the crash cart to obtain the epinephrine and manually draw up the drug from a vial. We know that epi auto-injectors are quite expensive, so if you would like to substitute either an expired one or a trainer, that is ok. Just switch it out at the last minute.

\section{Preparing for data collection during the simulation}

Please review the data collection form and demographics form so you will know what to look for as you observe the simulation.

1) Timed metrics that we are interested in:

- Time $0=$ when the healthcare team starts the simulation (typically when the nurse or practitioner goes to assess the patient) after they have been given the starting prompt by the simulation team.

- Time elapsed for the epinephrine to be ordered, even if the dose is not specified. 
- This time is for when the verbal order is given (not when it is entered into a computer).

- Some practitioners may just ask for "a dose of epi for anaphylaxis" but not give the dose until later when asked. Please record the earlier time but make a note that the dose was not specified. This will be a question in the online data collection tool.

- Time elapsed before the epinephrine is administered in relation to the start of the simulation.

It would be best if you can video record the simulation to ensure accuracy of these times. It does not have to be super high tech. I am planning on just using my iPhone to record this. For some of you, it may be that you use a stopwatch as your timer. After you have collected the timed metrics, you can delete the video. I do not need it for this study.

2) Try to position yourself close to the medication preparation area so that you can watch for any mistakes that are made. Did they choose the right dose and concentration of epi? Did they use the correct route of administration? If an auto-injector is used, did they know how to use the auto-injector correctly? The drug should be administered IM in the anterolateral thigh. For the auto-injectors, they should be held in place for at least 3 secs if not longer. (Please know that some healthcare staff have accidentally injected themselves when using auto-injectors. If you see that is about to happen, it would be ok to stop them.)

\section{The simulation scenario}

We purposely chose a very simple scenario because we wanted to make sure we could standardize it across sites. No confederate or parent needs to be involved or trained. We are not testing to see if participants can diagnose anaphylaxis. We want to see what they do to treat anaphylaxis.

\section{Equipment/Set Up}

1. 5 year old manikin (high of low fidelity) with a PIV in place.

2. Cardiorespiratory monitor for manikin.

Starting vitals: HR 155, CR flash, BP 88/30, RR 26, saturations $94 \%$, wheezing and increased WOB, normal mental status

Weight - $20 \mathrm{~kg}$

3. Epinephrine supplies that your institution would normally have available such as an Epi Auto-injector, code cart, drug tray, syringes and needles for IV, subcutaneous, and intramuscular dosing, etc. Try to use the equipment that is already present in the real clinical setting. 
4. Institutional reference or medication dosing cognitive aid, as particular to each site.

5. Smart phone or video recording device to capture timed metrics.

Script to tell participants:

"Please come evaluate this patient. He just received an IV antibiotic and is now covered in hives and is having trouble breathing. He has a history of anaphylaxis and he is in anaphylaxis now. He weighs $20 \mathrm{~kg} . "$

Remember to start video recording, or start your stop watch once you finish telling the simulation participants this and they being the simulation $=$ Time 0 .

Scenario End: Right after the epinephrine is administered, the vitals can return to a normal state.

\section{Debriefing}

You can use whatever debriefing style you prefer. Any obvious errors (ex. medication error) should be debriefed.

Let the team know what the objectives of the scenario were and give them the option of not participating in the study. This was the statement included in the IRB application:

"The anaphylaxis scenario you just participated is an attempt to gain a better understanding of how pediatric anaphylaxis is managed both in our institution and at others. Information regarding epinephrine dosing, preparation and administration was collected. Any video recording performed was to ensure accuracy of timed metrics and drug preparation. The video will be destroyed immediately after this data is obtained. Results will be analyzed as research data. No identifying information will be included in any report, abstract, or publication originating from this data. This study is voluntary, and you may choose to not participate at any time by simply notifying the simulation team."

Examples of questions you could use if relevant to their performance:

1. Tell me more about the strategies you used that helped you to administer the correct dose of epinephrine for anaphylaxis so quickly.

2. Is there anything you would do differently the next time this happens?

3. Did anything surprise you about this scenario? Were there any non-routine events? How were those managed? 
4. Epinephrine is a high-risk medication and there have been errors made by multiple healthcare providers because of confusion over the correct concentration/ dosing/ route of administration. For example, the 1:1000 concentration for anaphylaxis has been given via the IV route. This has resulted in significant patient side effects including arrhythmias and hypertension.

a. What do you think could have gone wrong in that case?

b. Have any of you had a similar experience with epinephrine before? Perhaps a near-miss where the error was caught before it reached the patient? 


\section{Table E2: Specific Latent Safety Threats}

- No Standard Operating Procedure is available for Epinephrine use in our ED's anaphylaxis response.

Table E1: Characteristics of sites ( $n=17)$ with epinephrine auto injectors (EAI)

\begin{tabular}{|r|c|c|}
\hline & N & $\%$ \\
\hline Locations where EAl is first line therapy (\% of sites with EAl available) & & \\
\hline Emergency Department & 5 & 29 \\
\hline Oncology inpatient unit & 5 & 29 \\
\hline Inpatient unit (Non-Oncology) & 2 & 12 \\
\hline Intensive Care Unit & 0 & 0 \\
\hline Operating Room & 0 & 0 \\
\hline Radiology & 7 & 41 \\
\hline Outpatient clinic & 3 & 18 \\
\hline Where EAls are stored (\% of sites with EAl available) & 2 & 12 \\
\hline High reaction areas & & \\
\hline Code cart & 1 & 6 \\
\hline Medication dispensing unit & 8 & 47 \\
\hline Code Go bag & 1 & 6 \\
\hline Unit-specific medication box & 7 & 41 \\
\hline
\end{tabular}




\begin{tabular}{|c|c|}
\hline & $\begin{array}{l}\text { - There are delays in administering Epi because nursing is unable to } \\
\text { draw up medications for a patient until the patient is registered in } \\
\text { our } 2 \text { computer systems. }\end{array}$ \\
\hline & $\begin{array}{l}\text { - Outdated anaphylaxis protocol exists involving use of Epi Pen, but } \\
\text { autoinjectors are no longer available at the hospital. }\end{array}$ \\
\hline \multirow{9}{*}{$\begin{array}{l}\text { Specific Process: } \\
\text { Cognitive Aid }\end{array}$} & - There is no anaphylaxis dosing of epinephrine on the code sheet. \\
\hline & $\begin{array}{l}\text { Dosing on anaphylaxis sheet is written in } \mathrm{mg} \text { (not } \mathrm{mL} \text { ), which caused } \\
\text { a delay in administration because nursing needed to calculate the } \\
\text { volume to be administered. }\end{array}$ \\
\hline & $\begin{array}{l}\text { - Nursing staff was not familiar with the dosing book available at } \\
\text { every crash cart. The nurse almost drew up the arrest dose of } \\
\text { epinephrine but at the last minute saw the heading entitled } \\
\text { "anaphylaxis." }\end{array}$ \\
\hline & - Inappropriate labelling of Epi for anaphylaxis on the code sheet. \\
\hline & $\begin{array}{l}\text { - Our pre-made code sheets do not have an anaphylaxis dose, so our } \\
\text { team saw the 1:1000 Epinephrine dose listed for ETT administration } \\
\text { and used this inappropriate dosing. }\end{array}$ \\
\hline & - IM dosing of epinephrine not on code sheets. \\
\hline & - Outdated cognitive aid recommending subcutaneous route. \\
\hline & $\begin{array}{l}\text { - Two cognitive aids present in same room but with different doses } \\
\text { listed for anaphylaxis. }\end{array}$ \\
\hline & $\begin{array}{l}\text { - The fellow used a PALS card which lists both IM and IV dosing (IV } \\
\text { use for hypotension) and he inappropriately chose IV. }\end{array}$ \\
\hline \multirow{4}{*}{ Knowledge Gap } & $\begin{array}{l}\text { Dosing for anaphylaxis was unknown. The wrong volume and } \\
\text { concentration were asked for making the order a correct 'dose' but } \\
\text { incorrect concentration and route of epinephrine administration. }\end{array}$ \\
\hline & $\begin{array}{l}\text { - There was inadequate knowledge of our hospital process for } \\
\text { anaphylaxis. }\end{array}$ \\
\hline & $\begin{array}{l}\text { Due to a recent shortage of epinephrine, the nursing staff has been } \\
\text { diluting 1:1,000 epinephrine to the } 1: 10,000 \text { concentration for } \\
\text { cardiac arrest. So they inadvertently diluted the anaphylaxis dose as } \\
\text { well. }\end{array}$ \\
\hline & - Staff not aware that Epi Pens were stocked in our PYXIS \\
\hline $\begin{array}{l}\text { Specific Process } \\
\text { Pyxis }\end{array}$ & $\begin{array}{l}\text { - Epi Pen and Epi Pen Jr. should be stocked in the PYXIS for all units. } \\
\text { However, not all units had Epi Pen Jr stocked and when stocked it } \\
\text { was not available for override in case of emergency. }\end{array}$ \\
\hline
\end{tabular}




\begin{tabular}{|c|c|}
\hline & $\begin{array}{l}\text { - There was a several minute delay in administration of Epinephrine } \\
\text { because the team did not know how to obtain the anaphylaxis kit } \\
\text { from the PYXIS. }\end{array}$ \\
\hline \multirow{4}{*}{$\begin{array}{l}\text { Equipment/ } \\
\text { Human Factors } \\
\text { Related }\end{array}$} & - Medical team was unable to find the Epi pen in timely manner. \\
\hline & $\begin{array}{l}\text { - Anaphylaxis kit had epinephrine ampule without filter needle, } \\
\text { causing a significant delay in care. }\end{array}$ \\
\hline & $\begin{array}{l}\text { - The two different concentrations of epi are in bags with warning } \\
\text { stickers. However, the bags look exactly the same which caused the } \\
\text { wrong concentration to be utilized. }\end{array}$ \\
\hline & $\begin{array}{l}\text { - Pediatric code cart (in a community ED) was not appropriately } \\
\text { stocked. Supplies had been previously removed for other patients } \\
\text { and not replaced. }\end{array}$ \\
\hline
\end{tabular}

